\title{
GOMMENTARY
}

\section{Addressing Adverse Childhood Experiences in Family Medicine: A Multigenerational Approach}

Lorraine M. McKelvey, PhD; Nicola Conners Edge, PhD

(Fam Med. 2020;52(4):243-5.)

doi: 10.22454/FamMed.2020.278217

A dverse childhood experiences (ACEs) are a set of traumatic events in childhood that have a strong dose-response association with a multitude of negative physical and mental health outcomes in adulthood, including conditions associated with early mortality. ${ }^{1,2}$ The prevalence of ACEs in the general population is staggering; $62 \%$ of adults in the United States report having experienced at least one adversity, and 38\% report having experienced two or more ACEs. ${ }^{3}$ Data for children aged birth to 17 years suggests onethird have experienced at least one adversity. ${ }^{4}$ Recent studies have documented the longitudinal impacts of ACEs on health into adolescence and adulthood, with impacts beginning as early as infancy. ${ }^{5,6}$

As health care providers increasingly seek ways to improve patient outcomes by addressing ACEs,${ }^{7,8}$ we offer two key considerations for family medicine practices. First, addressing ACEs through a trauma-informed care (TIC) approach involves organizational changes that should precede the initiation of screening and changes to clinical practice. Second, family medicine is uniquely positioned to reduce the impacts of ACEs and trauma for future generations because of screening protocols that are already in place.

Experts strongly recommend that organizational changes to create a more trauma-informed environment should precede changes in patient care. The first step to building TIC typically involves educating all clinical and nonclinical staff. In a TIC organization, all individuals must learn how trauma affects people and the steps to recovery, how to recognize signs of trauma and respond appropriately, and how to resist retraumatization of patients and staff. $^{9}$ Other foundational steps include engaging trauma survivors in the review of policies and procedures, ensuring a safe physical environment, and establishing a culture of staff wellness. ${ }^{9,10}$ It is important to acknowledge that many of us in the helping professions have experienced ACEs, too. ${ }^{11,12}$ Therefore, it is imperative for us to make considerations for the comfort of our staff and colleagues, in addition to our patients, as we work toward becoming trauma-informed.

In the context of a trauma-informed environment, we are more likely to realize the promise associated with screening for ACEs if we avoid potential pitfalls. Having ways to recognize trauma in patients and staff is a component of TIC. However, screening can only benefit the patient if it leads to more appropriate care. When ACEs are identified, symptom monitoring is critical, but rescreening should be avoided due to the risk of retraumatization. ${ }^{10}$ Therefore, screening for trauma in large health systems will take planning and careful thought. Because adult-reported ACEs are immutable, appropriate agreements for information sharing will be required. Further, the inclusion of ACEs in electronic health records will need to be carefully planned to avoid rescreening and insure proper use of the results across providers.

From the University of Arkansas for Medical Sciences, College of Medicine, Department of Family and Preventive Medicine, Little Rock, AR. 
In addition to becoming a safe place to inquire about trauma, there is also a need for proper response to identification. Unfortunately, a recent systematic review of primary care practices that implement widespread ACEs screening found that while the inquiry was generally acceptable to clinicians and patients, there was little change to clinical practice after identification. ${ }^{13}$ Protocols and relationships with integrated behavioral health providers and community partners should be developed to provide resources for those with ACEs. Staff will need support to provide or to make referrals to evidence-based treatments appropriate for patients with significant symptoms of posttraumatic stress disorder (PTSD). They will also need training in approaches that may be useful for patients without PTSD but who experience stress or engage in risky health behaviors. Examples of these approaches are motivational interviewing, mindfulness training, and peer support. ${ }^{10}$

While family physicians are working toward being trauma-informed and developing appropriate responses to ACEs screening for adults, we can capitalize on existing screening protocols by connecting results to consequences for their children. The ACEs index from the original CDC-Kaiser study includes 10 indicators. Five assess child maltreatment, and five reflect characteristics of the parent or home environment, including living in a home with mental illness, substance abuse, domestic violence, a history of incarceration, and separation or divorce. Many family physicians screen adult patients for depressive symptoms, substance use, and domestic violence. For children living in the home with those adults, these factors represent an ACE, and intervening immediately means an opportunity to reduce the impact of that ACE on the child's health and development.

Expanded use of depression screening and identification are also important. Family physicians implement screening approaches consistent with the recommendations of the American Academy of Pediatrics and the US Preventive Services Task Force for screening for maternal depression. ${ }^{14,15}$ Family medicine residents are trained to address depression, but there are opportunities to increase awareness about the impact of maternal depression on child well-being and about clinical approaches to support mothers and their children when this risk is present. Many options exist for supporting caregivers and their children when a risk like depression, substance use, or childhood trauma has been identified. ${ }^{16}$ In addition to motivational interviewing and referrals for behavioral health interventions, health professionals can provide referrals to external parenting supports such as home visiting and high-quality child care. ${ }^{17}$ In-house psychoeducation can be provided to teach the ways adults can support healthy child development. ${ }^{18}$ The IMPLICIT network has demonstrated that well-child visits in family medicine clinics can be used to identify and support high-risk mothers and children. ${ }^{19}$ All of these options hold potential for reducing a child's exposure to ACEs as well as for enhancing protective factors that reduce the likelihood of poor outcomes.

As multigenerational practitioners, family physicians have great potential to positively impact families across generations. Building a trauma-informed organization will take time. Meanwhile, we can act individually. Each of us can take small but important steps to build upon screening protocols and reduce the long-term impact of ACEs on the families we serve today.

CORRESPONDING AUTHOR: Address correspondence to Dr Lorraine McKelvey, University of Arkansas for Medical Sciences, College of Medicine, Department of Family and Preventive Medicine, 4301 W. Markham, Little Rock, AR 72205. mckelveylorraine@uams.edu.

\section{References}

1. Anda RF, Felitti VJ, Bremner JD, et al. The enduring effects of abuse and related adverse experiences in childhood. A convergence of evidence from neurobiology and epidemiology. Eur Arch Psychiatry Clin Neurosci. 2006;256(3):174-186.

2. Felitti VJ, Anda RF, Nordenberg D, et al. Relationship of childhood abuse and household dysfunction to many of the leading causes of death in adults. The Adverse Childhood Experiences (ACE) Study. Am J Prev Med. 1998;14(4):245258.

3. Centers for Disease Control and Prevention. Behavioral Risk Factor Surveillance System ACE Data. https://www.cdc.gov/ violenceprevention/childabuseandneglect/acestudy/ace-brfss. html. Accessed February 5, 2020.

4. Health Resources and Services Administration. National Survey of Children's Health Fact Sheet: October 2019. https://mchb.hrsa.gov/sites/default/files/mchb/Data/NSCH/ NSCH-2018-factsheet.pdf. Published 2019. Accessed October 21, 2019.

5. Widom CS, Horan J, Brzustowicz L. Childhood maltreatment predicts allostatic load in adulthood. Child Abuse Negl. 2015;47:59-69.

6. McKelvey LM, Saccente JE, Swindle TM. Adverse childhood experiences in infancy and toddlerhood predict obesity and health outcomes in middle childhood. Child Obes. 2019:15(3):206-215.

7. Sugimoto B. FPs Are Best Equipped to Tackle Adverse Childhood Experiences. AAFP Leader Voices Blog. https://www. aafp.org/news/blogs/leadervoices/entry/20200129lv-aces.html. Published January 29, 2020. Accessed February 5, 2020. 
8. Leasy M, O'Gurek DT, Savoy ML. Unlocking clues to current health in past history: childhood trauma and healing. Fam Pract Manag. 2019;26(2):5-10. https://www.aafp.org/ fpm/2019/0300/p5.html. Accessed February 3, 2020.

9. Substance Abuse and Mental Health Services Administration. SAMHSA's Concept of Trauma and Guidance for a Trauma-Informed Approach. HHS Publication No. (SMA) 14-4884. Rockville, MD: SAMHSA; 2014.

10. Menschner C, Maul A. Key ingredients for successful trauma-informed care implementation. Hamilton, NJ: Center for Health Care Strategies, Inc; 2016. https://www.chcs.org/ resource/key-ingredients-for-successful-trauma-informedcare-implementation/. Accessed March 2, 2020.

11. Strait J, Bolman T. Consideration of Personal Adverse Childhood Experiences during Implementation of TraumaInformed Care Curriculum in Graduate Health Programs. Perm J. 2017;21:16-061.

12. Sciolla AF, Wilkes MS, Griffin EJ. Adverse Childhood Experiences in Medical Students: implications for Wellness. Acad Psychiatry. 2019;43(4):369-374.

13. Ford K, Hughes K, Hardcastle K, et al. The evidence base for routine enquiry into adverse childhood experiences: A scoping review. Child Abuse Negl. 2019;91:131-146.
14. Siu AL, Bibbins-Domingo K, Grossman DC, et al; US Preventive Services Task Force (USPSTF). Screening for depression in adults: US preventive services task force recommendation statement. JAMA. 2016;315(4):380-387.

15. Committee on Practice and Ambulatory Medicine Bright Futures Periodicity Schedule Workgroup. 2017 Recommendations for Preventive Pediatric Health Care. Pediatrics. 2017;139(4):e20170254.

16. Substance Abuse and Mental Health Services Administration. Depression in Mothers: More Than the Blues-A Toolkit for Family Service Providers. HHS Publication No. (SMA) 14-4878. Rockville, MD: Substance Abuse and Mental Health Services Administration, 2014.

17. Edge NAC, Kraleti S, McKelvey L, Jarrett DM, Sublett JD II, Bennett IM. Training residents in maternal depression care to improve child health: a CERA study. Fam Med. 2019;51(9):756-759.

18. Trussell TM, Ward WL, Conners Edge NA. The impact of maternal depression on children: a call for maternal depression screening. Clin Pediatr (Phila). 2018;57(10):1137-1147.

19. Srinivasan S, Schlar L, Rosener SE, et al. Delivering interconception care during well-child visits: an IMPLICIT network study. J Am Board Fam Med. 2018;31(2):201-210. 